\title{
Long-Term Oral Toxicity and Anti-osteoporotic Effect of Sintered Dicalcium Pyrophosphate in Rat Model of Postmenopausal Osteoporosis
}

\author{
Yuh-Feng Tsai ${ }^{1,2} \cdot \mathrm{Li}-\mathrm{Ho} \mathrm{Hsu}^{3,4} \cdot$ Chang-Chin $\mathrm{Wu}^{3,4,5} \cdot$ Wei-Hua Cai $^{6}$ • \\ Kai-Chiang Yang ${ }^{6} \cdot$ Fang-Yu Fan ${ }^{6}$
}

Received: 12 August 2015/Accepted: 7 April 2016/Published online: 3 January 2017

(C) Taiwanese Society of Biomedical Engineering 2017

\begin{abstract}
Sintered dicalcium pyrophosphate (SDCP), a synthetic pyrophosphate analog, has shown potential for the management of osteoporosis. The long-term oral toxicity and anti-osteoporotic effect of SDCP in a postmenopausal osteoporosis rat model were evaluated in this study. SDCP was orally administered to bilateral ovariectomized (OVX) Wistar rats at a dose of $0.75 \mathrm{mg} / \mathrm{kg}$ daily for 24 weeks following by 2 weeks of observation. There were no abnormal findings in clinical signs of toxicity, food consumption, body weight, blood examination, necropsy, and histological inspection attributable to the ingestion of SDCP. The serum level of type I collagen fragments, a bone resorption marker, decreased in SDCP-treated rats, and the bone formation markers alkaline phosphatase, osteocalcin, and osteopontin significantly decreased. These
\end{abstract}

Kai-Chiang Yang

pumpkin@tmu.edu.tw

Fang-Yu Fan

fish884027@tmu.edu.tw

1 Department of Diagnostic Radiology, Shin-Kong Wu Ho-Su Memorial Hospital, Taipei City 111, Taiwan

2 School of Medicine, Fu-Jen Catholic University, New Taipei City 24205, Taiwan

3 Department of Orthopedics, En Chu Kong Hospital, New Taipei City 23702, Taiwan

4 Department of Orthopedics, College of Medicine, National Taiwan University Hospital, National Taiwan University, Taipei City 10002, Taiwan

5 Department of Biomedical Engineering, Yuanpei University of Medical Technology, Hsinchu 30015, Taiwan

6 School of Dental Technology, College of Oral Medicine, Taipei Medical University, $250 \mathrm{Wu}$-Hsing Street, Taipei City 11031, Taiwan findings indicate that the bone turnover rate decreased in SDCP-treated animals. Relative to OVX rats, the increase in serum tartrate-resistant acid phosphatase 5b level represents an increase in bony tissues in the SDCP-treated rats. Histological examinations of distal femoral metaphyses further revealed that the ingestion of SDCP improved the trabecular bone architecture and decreased bone porosity. Analysis of limb bone ashes showed a significant increase in bone mineral content. Our results show that SDCP inhibits bone resorption to restore bone mass in OVX rats without deleterious effects, and therefore that SDCP has potential in the management of osteoporosis.

Keywords Osteoporosis · Pyrophosphate analog · Sintered dicalcium pyrophosphate - Oral toxicity - Bone turnover markers

\section{Introduction}

Osteoporosis, frequently found in the elderly and postmenopausal women, is characterized by low bone mass, deteriorated bone architecture, and alterations in bone quality [1]. Bone loss results from bone resorption by osteoclasts exceeding bone formation by osteoblasts, and is generally accompanied by an increased bone turnover rate [2]. This progressive skeletal disease increases susceptibility to fractures, and thus causes high morbidity and mortality.

Bisphosphonates, synthetic pyrophosphate analogs, have physicochemical effects similar to those of pyrophosphates [3]. Bisphosphonates can modulate osteoclasts and promote apoptosis to decrease bone turnover rate $[4,5]$. The binding of the bisphosphonate moiety to bone mineral inhibits osteoclast activity and limits the recruitment of 
osteoclasts to the bone surface [6]. Therefore, bisphosphonates can be used to treat Paget's disease, tumor-induced hypercalcemia, tumor-associated osteolysis, and osteoporosis [7]. The primary side effect of bisphosphonates is dose-dependent upper gastrointestinal irritation and esophageal ulcers [8]. A previous study reported bisphosphonate-associated osteonecrosis of the jaw [9]. Reid et al. reported adverse events in patients who had received intravenous zoledronate (ZLN) treatment [10]. Similarly, Black et al. and Lyles et al. found significantly high adverse event rates in patients receiving bisphosphonate treatment compared to those of a placebo group [11, 12]. Likewise, post-marketing reports from healthcare authorities show renal impairment associated with ZLN treatments. Thus, a new anti-osteoporosis regimen is necessary.

Beta-calcium pyrophosphate is an intermediate product in the biological mineralization process [13]. Pyrophosphate has recently been reported to be an osteoconductive material [14]. A pyrophosphate analog, sintered dicalcium pyrophosphate (SDCP), may have better biocompatibility than that of bisphosphonates. SDCP was first used as a biodegradable bone graft substitute, and histological examinations of rabbits receiving SDCP implantation in femoral condyles showed new bone deposition on the material surface [15]. Sun et al. studied the biocompatibility of a series of calcium phosphates. They reported that hydroxyapatite inhibited the growth of osteoblasts, and that SDCP showed better biocompatibility to osteoblasts [16]. Osteoblasts were treated with the ionic products of SDCP dissolution and the gene-expression pattern was determined [17]. The osteoblasts cultured in a medium with SDCP dissolution had a faster proliferation rate than that of cells cultured in a normal medium. In addition, the mRNA expression of osteocalcin was up-regulated, whereas the mRNA levels of type 1 collagen, osteonectin, and osteopontin were down-regulated. Furthermore, ultrastructural examinations of osteoclasts have shown SDCP-induced osteoclast apoptosis in terms of nuclei changes and cellular organelle degradation [18]. More recently, SDCP was shown to decrease the bone turnover rate and to promote fracture healing in a rat femoral fracture model [19]. Taken together, these studies suggest that SDCP may be used in the management of osteoporosis.

Ovariectomized (OVX) animals are a convenient and reliable model of postmenopausal osteoporosis [20]. Previous acute and subacute oral toxicity studies have shown that the ingestion of SDCP did not have any deleterious effect on OXV rats. Furthermore, oral administration of SDCP can decrease bony porosity and increase bone mineral content [21]. The purpose of the present study was to further evaluate the long-term oral toxicity of SDCP and the effects of SDCP on bone turnover markers and bone mass in osteoporotic rats.

\section{Materials and Methods}

\subsection{Experimental Animals and Oral Administration}

A total of forty female Wistar rats (obtained from the National Taiwan University Hospital Laboratory Animal Center), aged 10-11 weeks and weighing $193 \pm 18 \mathrm{~g}$, were used. Animals were maintained in accordance with the guidelines for the care and use of laboratory animals. Experimental protocols and surgical procedures were approved by the Institutional Animal Care and Use Committee. Animals were marked to permit individual identification, and kept in cages for 2 weeks before the beginning of experiments to allow for acclimatization to the laboratory environments.

The rats were randomly divided into four groups [sham, OVX, OVX + SDCP, and OVX + alendronate (ALN)], with 10 rats in each group. For the sham group, the rats were subjected to a sham operation without ovariectomy. The rats in the other three groups underwent ovariectomy via a lateral retroperitoneal approach. SDCP (Purzer Pharmaceutical Co. Ltd., Taiwan) was administered from 4 weeks after surgery. For the sham and OVX groups, the rats were treated with vehicle (phosphate buffered saline; PBS) by gavage with a stomach tube. For the OVX + SDCP group, the rats were treated with SDCP $(0.75 \mu \mathrm{g} / \mathrm{kg}$ body weight, suspended in PBS) daily by gavage in the early morning after $12 \mathrm{~h}$ of starvation. For the OVX + ALN group, the rats received ALN daily at a dose of $1.0 \mu \mathrm{g} / \mathrm{kg}$ body weight. The rats received PBS or the test compounds orally for 24 weeks followed by 2 weeks of observation. The body weights of all rats were measured twice a week.

\subsection{Clinical Signs}

We observed the clinical signs of toxicity twice a day (preand post-oral administration) throughout the dosing period, and once a day during the observation period. The rats were observed individually after oral administration during the first $30 \mathrm{~min}$, with special attention being paid during the first $4 \mathrm{~h}$ thereafter. When an animal continued to display signs of toxicity, additional observations were made and systematically recorded. Animals found in a moribund condition, showing severe pain, or enduring signs of severe distress were humanely sacrificed, and the time of death was recorded. The body weights of all rats and the gross weights of the feeders were determined twice a week.

\subsection{Blood Examination}

Blood samples were collected through intracardiac puncture immediately following sacrifice on the day after the observation period, and blood samples were analyzed 
within $24 \mathrm{~h}$ (by the National Taiwan University Veterinary Hospital). For hematology, erythrocyte count (red blood cells, RBCs), hemoglobin concentration ( $\mathrm{Hb})$, hematocrit value $(\mathrm{Ht})$, mean corpuscular volume (MCV), mean corpuscular hemoglobin (MCH), mean corpuscular hemoglobin concentration (MCHC), and leukocyte count (white blood cells, WBCs) were analyzed. For blood biochemistry, albumin, alanine aminotransferase (ALT/GPT), aspartate aminotransferase (AST/GOT), blood urea nitrogen (BUN), creatinine (CRE), glucose, total protein (TP), albumin, calcium $\left(\mathrm{Ca}^{+}\right)$, and inorganic phosphorus (IP) were analyzed.

\subsection{Serum Level of Bone Turnover Markers}

Sera were separated from whole blood samples and stored at $-80{ }^{\circ} \mathrm{C}$ until analysis. Bone turnover markers including osteocalcin (OC, Rat-MID ${ }^{\mathrm{TM}}$ Osteocalcin EIA, Immunodiagnostic Systems Limited, Boldon, UK), osteopontin (OPN, Rat Osteopontin Assay Kit, Immuno-Biological Laboratories, Gunma, Japan), tartrate-resistant acid phosphatase 5b (TRACP-5b, RatTRAP ${ }^{\mathrm{TM}}$ Assay, Immunodiagnostic Systems Limited), fragments of type I collagen (CTX-1, RatLaps ${ }^{\text {TM }}$ EIA, Immunodiagnostic Systems Limited), and intact parathyroid hormone (PTH, Intact PTH EIA, Alpco Diagnostics, Salem, NH, USA) were evaluated with enzyme-linked immunosorbent assays. The results of the assays were determined using a microplate reader (Multiskan ${ }^{\mathrm{TM}}$ GO Microplate Photometer, Thermo, USA) at wavelengths of $450 \mathrm{~nm}$ (for CTX-1, OC and PTH) and $405 \mathrm{~nm}$ (for TRACP-5b and OPN). The serum alkaline phosphatase (ALP) level was analyzed by the National Taiwan University Veterinary Hospital.

\subsection{Histological Examination of Visceral Organs}

After sacrifice, the major visceral organs such as the heart, liver, spleen, lung, stomach, and kidney were removed carefully for gross examinations. The samples were then fixed in neutral buffered $10 \%$ formalin solution for histological study. The samples were dehydrated using a graded ethanol series and embedded in paraffin wax. Consecutive sections were cut from the paraffin blocks into $5-\mu \mathrm{m}$ slices and mounted onto slides. Sections were stained with hematoxylin and eosin (H\&E, 3008-1\&3204-2, Muto, Japan) and examined using an optical microscope.

We also harvested the long bones of the four limbs (femur, tibia, humerus, and forearm). The soft tissues on long bones were trimmed, cleaned, and preserved for further analysis.

\subsection{Evaluations of Femurs}

A digital caliper was used to measure the length and width of the femurs (lateral dimension). Archimedes' principle was used to determine the bone volume of harvested long bones.

After the measurements, the harvested femurs were decalcified using decalcification agents and the osteotomized samples were prepared for histological inspections. Sections were stained with Goldner's trichrome stain (Muto Pure Chemicals, Tokyo, Japan). The sections of distal femur metaphyses were used to evaluate the bony porosity, and the thickness and interconnections between trabeculations were evaluated as done in a previous study [21]. Five non-continuous sections were observed for each sample, and five rats were included to calculate the bony porosity for each group.

\subsection{Analysis of Bone Ash}

The other long bones of the rats were dried in an oven set at $60{ }^{\circ} \mathrm{C}$ for 3 days. The dry weight of each long bone was then measured $\left(\mathrm{w}_{\mathrm{i}}\right)$, and finally the bones were burned in a furnace set at $800{ }^{\circ} \mathrm{C}$ for $1 \mathrm{~h}$. The weight of the bone ash was measured $\left(\mathrm{w}_{\mathrm{f}}\right)$, and the ratio of the weight of the bone ash to the dry weight $\left(\mathrm{w}_{\mathrm{f}} / \mathrm{w}_{\mathrm{i}}\right)$ was calculated to determine the bone mineral content.

\subsection{Statistical Analysis}

The data obtained from each group are expressed as mean \pm standard error of the mean (SEM). Analysis of variance was used with post hoc Dunnett's multiple comparison tests to analyze the differences between groups. A difference was considered statistically significant if the $p$ value was smaller than 0.05 .

\section{Results}

\subsection{Clinical Sign of Toxicity}

All rats were observed individually after ingesting SDCP every day during the 24-week dosing period and an additional 2-week observational period. Observations included changes in skin and fur, eyes, mucous membrane, respiratory, circulatory, autonomic, and central nervous systems, somatomotor activity, and behavior patterns. Special attention was paid to observations of tremors, convulsions, salivation, diarrhea, lethargy, sleep, and coma. There were no abnormal clinical signs of toxicity in the OVX + SDCP and $\mathrm{OVX}+\mathrm{ALN}$ groups during the long-term oral 
toxicity study, and all of the rats showed normal vitality. No rat was found dead during the dosing or observational periods.

\subsection{Body Weight Change and Food Consumption}

The body weight of the rats in each group increased with time (Fig. 1). No significant differences were noticed between the OVX + SDCP and OVX + ALN groups. However, the body weights of these two dosing groups were higher than that of the sham group and lower than that of the OVX group. Food consumption was normal, and there were no obvious differences between these four groups.

\subsection{Blood Examination}

There were no changes attributable to the test substances with regards to hematology for all tests (Table 1). After ingestion of SDCP for 24 weeks, the biochemical parameters in sera including albumin, ALT/GPT, AST/GOT, BUN, CRE, glucose, TP, $\mathrm{Ca}^{+}$, and IP did not show any significant changes with regards to long-term oral toxicity.

\subsection{Serum Level of Bone Turnover Markers}

Statistically significant changes in the bone turnover markers were noticed among groups. After ovariectomy, the serum ALP level increased significantly from $94.37 \pm 28.11 \mathrm{U} / 1$ in the sham group to $348.30 \pm 65.69$ $\mathrm{U} / 1$ in the OVX group $(p<0.001$, Fig. 2a). Ingestion of SDCP decreased the ALP level to $167.34 \pm 48.31 \mathrm{U} / \mathrm{l}$ in the OVX + SDCP group $(p<0.01$ compared with OVX group), and the ALP level was $123.14 \pm 38.24 \mathrm{U} / 1$ in the OVX + ALN group $(p<0.01$ compared with OVX group). No significant difference was found in the ALP level between OVX + SDCP and OVX + ALN groups.

The serum OC level increased from $13.89 \pm 4.01 \mathrm{ng} / \mathrm{ml}$ in the sham group to $32.74 \pm 4.12 \mathrm{ng} / \mathrm{ml}$ in the OVX group $(p<0.01$, Fig. $2 b)$. In contrast, the OC level decreased to $22.26 \pm 5.33 \mathrm{ng} / \mathrm{ml}$ in the OVX rats administered with $\operatorname{SDCP}(p<0.05$, compared with OVX group). The OC level was $25.94 \pm 3.86 \mathrm{ng} / \mathrm{ml}$ in the OVX + ALN group ( $p<0.05$ compared with OVX group). There were no significant differences in the OC level between OVX + SDCP and OVX + ALN groups. However, the OVX + ALN group had a significantly higher OC level relative to that of the sham group $(p<0.05)$.

The OPN level increased from $18.10 \pm 4.70 \mathrm{ng} / \mathrm{ml}$ in the sham group to $45.33 \pm 5.16 \mathrm{ng} / \mathrm{ml}$ in the OVX group $(p<0.01$, Fig. 2c). The OPN level decreased to $29.24 \pm 4.69 \mathrm{ng} / \mathrm{ml}$ in the OVX + SDCP group $(p<$ 0.05 , compared with OVX group) and $25.57 \pm 5.39 \mathrm{ng} / \mathrm{ml}$ in the OVX + ALN group $(p<0.05$, compared with OVX group). Between OVX + SDCP and OVX + ALN groups, no significant difference was found in the OPN level. The OVX + SDCP group had a significantly higher OPN level when compared with that of the sham group $(p<0.05)$.

The serum TRACP-5b level decreased from $6.18 \pm 1.67 \mathrm{U} / 1$ in the sham group to $1.93 \pm 0.89 \mathrm{U} / 1$ in the OVX group ( $p<0.01$, Fig. $2 d)$. The TRACP-5b level increased to $3.20 \pm 1.43 \mathrm{U} / \mathrm{l}$ in the $\mathrm{OVX}+\mathrm{SDCP}$ group $(p<0.05$, compared with OVX group) and $4.22 \pm 1.23$ $\mathrm{U} / \mathrm{l}$ in the OVX $+\mathrm{ALN}$ group $(p<0.05$, compared with OVX group). There were no significant differences in the TRACP-5b level between OVX + SDCP and OVX +
Fig. 1 Body weight changes of rats. There was no significant difference between

OVX + SDCP and

OVX + ALN groups. Body weights of these two groups were heavier than that of sham group and lower than that of OVX group

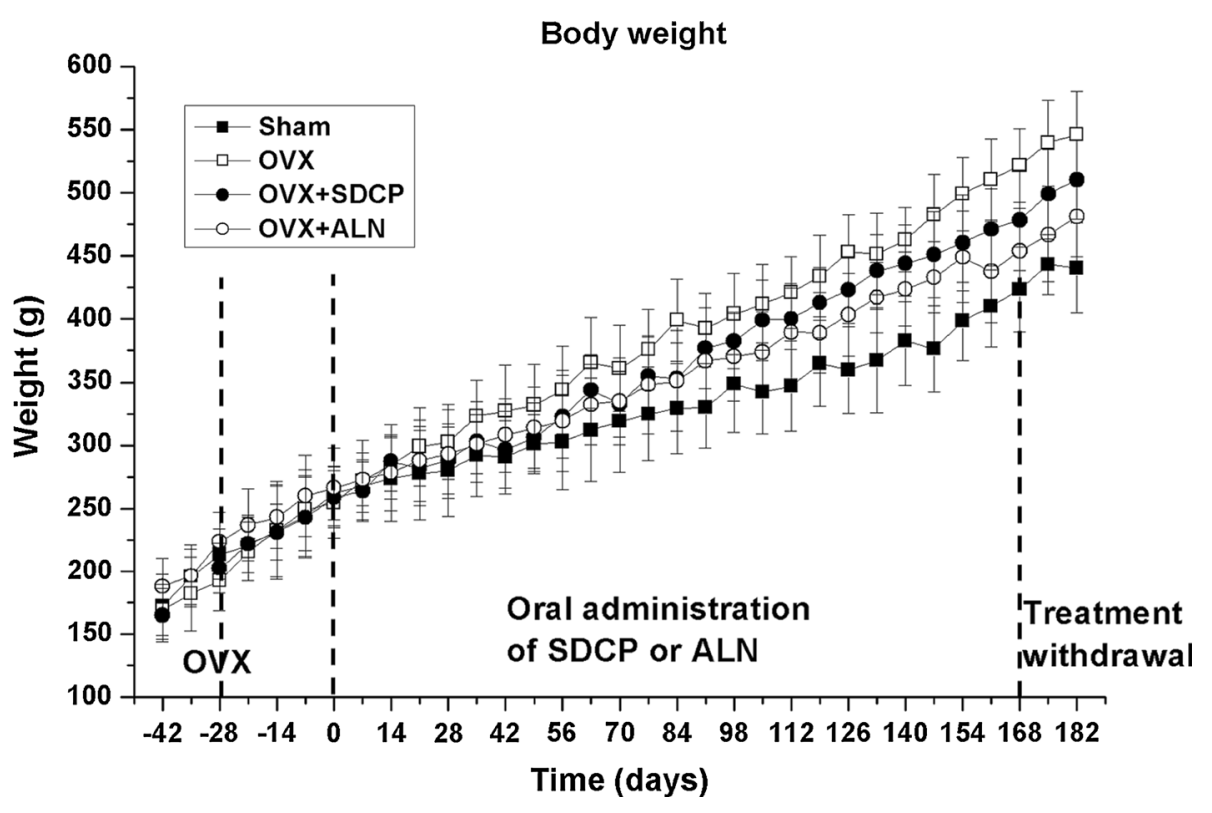


Table 1 Hematological and blood biochemical analyses of long-tern oral toxicity study

\begin{tabular}{|c|c|c|c|c|c|}
\hline & & Sham & OVX & $\mathrm{OVX}+\mathrm{SDCP}$ & $\mathrm{OVX}+\mathrm{ALN}$ \\
\hline RBC & $\times 10^{6} / \mu 1$ & $8.5 \pm 0.8$ & $7.8 \pm 0.4$ & $8.6 \pm 0.8$ & $8.0 \pm 0.6$ \\
\hline $\mathrm{Hb}$ & $\mathrm{g} / \mathrm{dl}$ & $14.9 \pm 1.2$ & $14.0 \pm 0.5$ & $14.8 \pm 1.0$ & $15.0 \pm 1.3$ \\
\hline $\mathrm{Ht}$ & $\%$ & $44.7 \pm 2.1$ & $39.3 \pm 1.4$ & $42.2 \pm 4.6$ & $40.0 \pm 2.6$ \\
\hline $\mathrm{MCV}$ & $\mathrm{Fl}$ & $52.9 \pm 0.6$ & $50.3 \pm 1.4$ & $49.0 \pm 2.1$ & $50.4 \pm 1.0$ \\
\hline $\mathrm{MCH}$ & $\mathrm{Pg}$ & $16.4 \pm 1.3$ & $19.1 \pm 0.7$ & $17.3 \pm 2.1$ & $18.7 \pm 0.7$ \\
\hline MCHC & $\%$ & $36.4 \pm 0.9$ & $38.0 \pm 1.1$ & $35.2 \pm 4.0$ & $37.1 \pm 1.7$ \\
\hline WBC & $/ \mu \mathrm{l}$ & $7267 \pm 943$ & $8250 \pm 1280$ & $7150 \pm 1034$ & $6670 \pm 1454$ \\
\hline Albumin & $\times 10^{4} / \mu 1$ & $4.3 \pm 0.3$ & $3.9 \pm 0.2$ & $4.5 \pm 0.4$ & $4.6 \pm 0.4$ \\
\hline ALT/GPT & $\mathrm{g} / \mathrm{dl}$ & $57.7 \pm 11.5$ & $68.8 \pm 8.7$ & $49.7 \pm 19.3$ & $89.3 \pm 14.2$ \\
\hline AST/GOT & $\mathrm{U} / 1$ & $116 \pm 43$ & $106 \pm 39$ & $139 \pm 57$ & $122 \pm 53$ \\
\hline BUN & $\mathrm{U} / \mathrm{l}$ & $16.3 \pm 1.5$ & $19.3 \pm 2.4$ & $17.3 \pm 0.9$ & $20.8 \pm 1.7$ \\
\hline CRE & $\mathrm{U} / 1$ & $0.7 \pm 0.1$ & $0.5 \pm 0.1$ & $0.6 \pm 0.1$ & $0.7 \pm 0.1$ \\
\hline Glucose & $\mathrm{mg} / \mathrm{dl}$ & $103 \pm 28$ & $110 \pm 21$ & $105 \pm 26$ & $120 \pm 37$ \\
\hline $\mathrm{TP}$ & $\mathrm{mg} / \mathrm{dl}$ & $7.8 \pm 0.7$ & $7.4 \pm 0.8$ & $7.0 \pm 0.6$ & $7.4 \pm 0.4$ \\
\hline $\mathrm{Ca}^{+}$ & $\mathrm{mg} / \mathrm{dl}$ & $8.2 \pm 1.1$ & $9.3 \pm 1.8$ & $11.0 \pm 0.9$ & $10.4 \pm 1.0$ \\
\hline IP & $\mathrm{g} / \mathrm{dl}$ & $7.4 \pm 1.1$ & $8.2 \pm 0.5$ & $8.2 \pm 1.3$ & $8.7 \pm 1.3$ \\
\hline
\end{tabular}

a
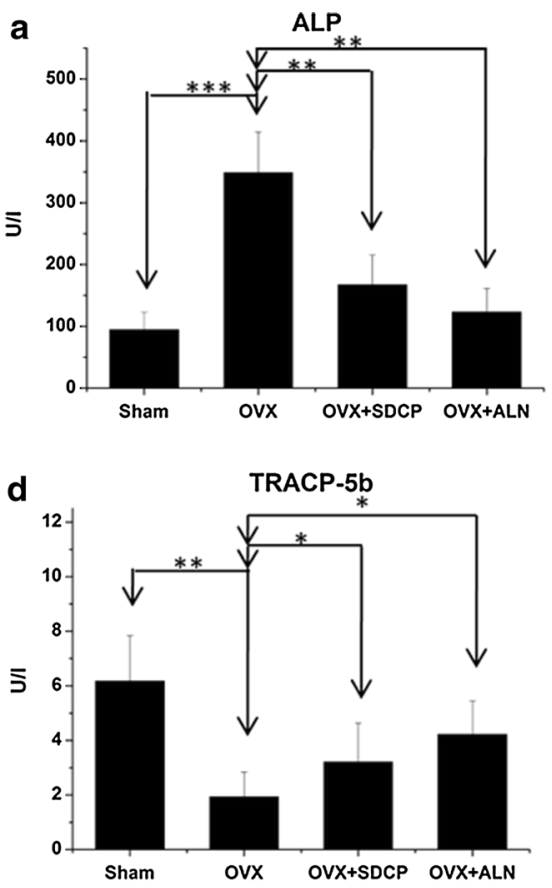

b
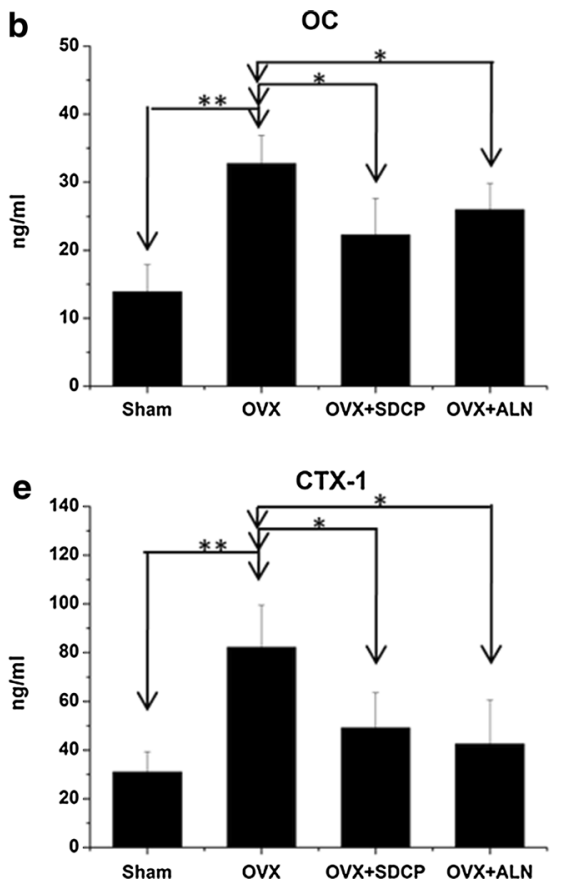

C
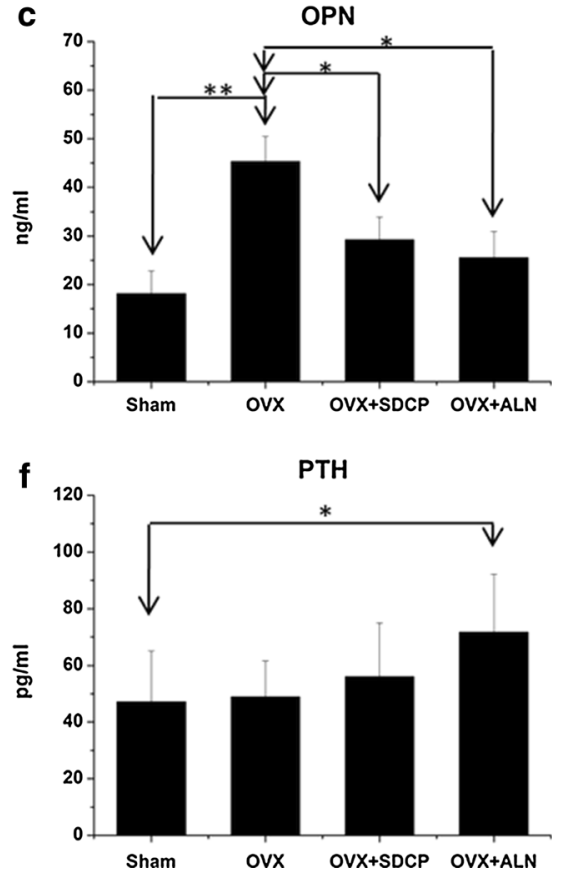

Fig. 2 After ovariectomy, serum levels of bone formation markers a ALP, b OC, and $\mathbf{c}$ OPN in OVX group increased significantly. d Level of TRACP-5b significantly decreased in OVX group. e CTX-1 also increased in OVX group. In contrast, administration of SDCP significantly decreased levels of ALP, OC, OPN, and CTX-1, but increased level of TRACP-5b. Similar findings were observed in OVX + ALN group. f Serum level of intact PTH increased in OVX + ALN group. There were no significant differences among sham, OVX, and OVX + SDCP groups

ALN groups. However, the OVX + SDCP group had a significantly lower TRACP-5b level when compared with that of the sham group $(p<0.05)$.

The level of CTX-1 increased from $30.95 \pm 8.38 \mathrm{ng} / \mathrm{ml}$ in the sham group to $82.16 \pm 17.28 \mathrm{ng} / \mathrm{ml}$ in the OVX group ( $p<0.01$, Fig. 2e). The CTX-1 level decreased to
$49.13 \pm 14.43 \mathrm{ng} / \mathrm{ml}$ in the OVX + SDCP group ( $p<0.05$, compared with OVX group) and $42.55 \pm$ $18.04 \mathrm{ng} / \mathrm{ml}$ in the OVX + ALN group ( $p<0.05$, compared with OVX group). There were no significant differences in the CTX-1 level among sham, OVX + SDCP, and $\mathrm{OVX}+\mathrm{ALN}$ groups. 
The serum level of intact PTH was $47.14 \pm 17.88 \mathrm{pg} /$ $\mathrm{ml}$ in the sham group and $48.79 \pm 12.64 \mathrm{pg} / \mathrm{ml}$ in the OVX group (Fig. 2f). There were no significant differences between these two groups. The PTH level increased to $71.68 \pm 20.49 \mathrm{pg} / \mathrm{ml}$ in the OVX + ALN group. There were no significant differences in the PTH level among sham, OVX, and OVX + SDCP groups. The OVX + ALN group had a significantly higher PTH level when compared with sham and OVX groups (both $p<0.05)$.

\subsection{Histological Examination of Visceral Organs}

For the major visceral organs, the histological sections of heart tissue revealed that the cells were lined in parallel, had an equal size, and there were no pathological findings. There was no focal myocardial degeneration or fibrosis. The liver sections showed that the hepatocytes were arranged normally in a single column without other remnant cells. No microgranulomas or focal necrosis was found. The histological findings of the kidney sections showed that the glomerulus, Bowman's capsule, renal tubules, and renal corpuscles were normal, without abnormal fibrosis. There were no cysts, pelvic dilatation, hyaline droplets in the proximal urinary tubular epithelium, or focal interstitial infiltration of lymphocytes in the kidneys. The histological findings of the spleen sections were normal, without abnormal growth of B or $\mathrm{T}$ lymphocytes. The lung sections showed normal epithelium cells in the alveoli. There were no changes in parietal cells or gastric glands. All histological sections appeared normal in the long-term oral toxicity study of SDCP.

\subsection{Effect of SDCP on Femurs}

No significant difference was found in bone volume, length, width, and dry weight between OVX and sham groups (Table 2). There was no significant difference in all tested parameters in any of the dosing groups compared with the OVX group. There was also no significant difference between dosing groups and the sham group.

In the histological study of the femurs of the sham group, the cancellous bone showed intervening trabecular bone with connectivity of the trabeculae elements (Fig. 3a). In OVX rats, there was thinning and disconnection of the trabeculae in the femurs compared with the sham group (Fig. 3b). Sections of the OVX + SDCP (Fig. 3c) and OVX + ALN (Fig. 3d) groups showed an improved bony architecture, and the femurs showed dense trabecular bones with partial restoration of interconnection. The porosity of bony trabeculae also increased significantly in the OVX group ( $p<0.01$, compared with sham group). After ingestion of SDCP or ALN, the porosity of bony trabeculae in the osteoporotic rats decreased, and there were increases in the trabeculation of femurs compared with the OVX group. The porosity decreased to $65.97 \pm 5.88 \%$ in the OVX + SDCP group and to $62.59 \pm 7.57 \%$ in the OVX + ALN group (Table 2). The porosities of OVX + SDCP and OVX + ALN groups were significantly lower than that of the OVX group $(p<0.01)$ but still higher than that of the sham group $(p<0.05)$. No significant difference was found in porosity between OVX + SDCP and OVX + ALN groups.

\subsection{Bone Ash Analysis}

After ovariectomy (OVX group), the ratio of the weight of the bone ash to the dry weight $\left(\mathrm{w}_{\mathrm{f}} / \mathrm{w}_{\mathrm{i}}\right)$ decreased significantly (Fig. 4a: humerus, 4b: forearm, 4c: femur, and $4 \mathrm{~d}$ : tibia). The ingestion of SDCP significantly increased the $\mathrm{w}_{\mathrm{f}} / \mathrm{w}_{\mathrm{i}}$ ratio compared with that of the OVX group $(p<0.05$ for all limbs), and ALN also significantly increased the $\mathrm{w}_{\mathrm{f}} /$ $\mathrm{w}_{\mathrm{i}}$ ratio $(p<0.05$ for all limbs). No significant difference was found in the $\mathrm{w}_{\mathrm{f}} / \mathrm{w}_{\mathrm{i}}$ ratios of the humerus and forearm among sham, OVX + SDCP, and OVX + ALN groups. However, the $\mathrm{w}_{\mathrm{f}} / \mathrm{w}_{\mathrm{i}}$ ratios of the femur and tibia of the OVX + SDCP group were significantly lower than those of the OVX + ALN group (both $p<0.05$ ).

\section{Discussion}

SDCP, which can reduce bone resorption, should be useful for the treatment of osteoporosis [22]. Although previous acute and subacute oral toxicity studies have reported that the ingestion of SDCP did not show deleterious effects on test subjects [21], the long-term oral toxicity has not been tested. Regarding the potential toxic effects of SDCP, we found no clinical signs of toxicity and no changes in food
Table 2 Effects of SDCP and ALN treatment on bone size and weight of femur

\begin{tabular}{llcccc}
\hline & & Sham & OVX & OVX + SDCP & OVX + ALN \\
\hline Length & $\mathrm{mm}$ & $44.23 \pm 1.01$ & $42.87 \pm 1.14$ & $43.18 \pm 1.13$ & $43.79 \pm 0.92$ \\
Width & $\mathrm{mm}$ & $3.81 \pm 0.38$ & $3.84 \pm 0.36$ & $3.81 \pm 0.38$ & $3.93 \pm 0.33$ \\
Volume & $\mu \mathrm{l}$ & $596 \pm 26$ & $607 \pm 43$ & $629 \pm 38$ & $637 \pm 49$ \\
Porosity (distal femur) & $\%$ & $59.10 \pm 4.66$ & $76.05 \pm 9.94$ & $65.97 \pm 5.88$ & $62.59 \pm 7.57$ \\
\hline
\end{tabular}



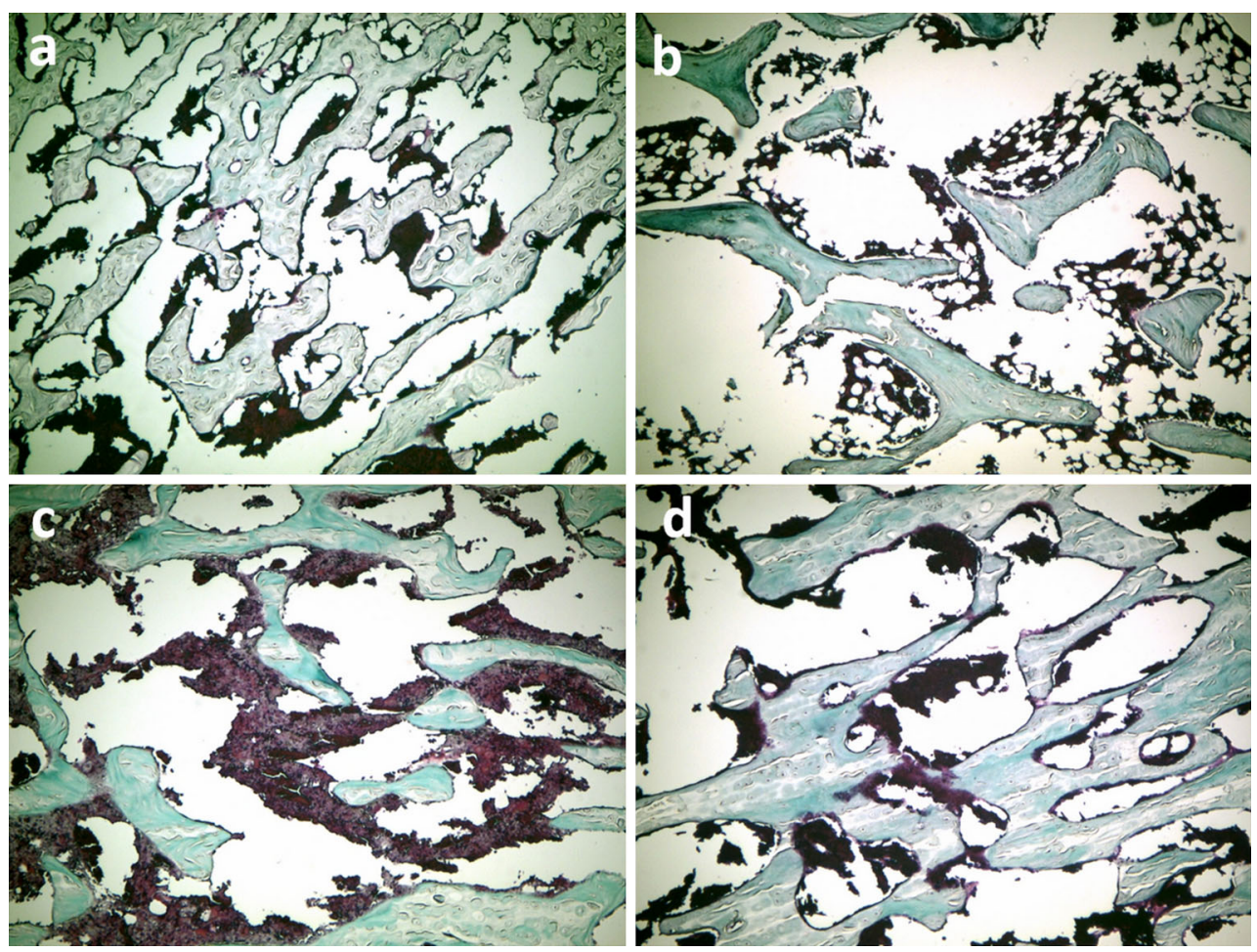

Fig. 3 a Histological sections (Goldner's trichrome stain) of distal femur showed intervening trabecular bone with connectivity of trabeculae elements in sham group. b After ovariectomy (OVX group), there was significant thinning and disconnection of trabeculae in the femur compared with sham group. c Administration of SDCP and d ALN partial restored bony architecture of healthy cancellous bone

consumption, hematology, or necropsy attributable to the oral administration of SDCP. The physiological conditions of the rats that received SDCP were normal and the animals were full of vim and vigor. In addition, the ingestion of SDCP did not have any deleterious effects on the major visceral organs when the histological sections were examined. These results were further verified by the findings of blood biochemical analysis.

To investigate possible hepatotoxicity with the longterm ingestion of SDCP, we analyzed AST/GOT and ALT/ GPT, two intracellular enzymes of hepatocytes. The blood biochemistry results showed no changes in the serum levels of either compared to the sham group or OVX rats. With regards to nephrotoxicity, BUN and creatinine are two indicators of the state of the kidney. The results also showed no changes in either compared with the sham group or OVX rats, suggesting that the ingestion of SDCP does not cause hepatotoxicity or nephrotoxicity.

Regarding the influence of SDCP ingestion on body weight, the OVX rats had a significantly greater weight gain than that of the sham group, which may be attributable to estrogen deficiency post-ovariectomy [23]. In contrast, the weight gain was slowed in the OVX + SDCP and OVX + ALN groups relative to the OVX group. It was reported that elcatonin has an inhibitory effect on appetite, and a similar finding was also observed in rats treated with ALN [24]. Previous acute and subacute oral toxicity studies showed that rats treated with SDCP had a relatively lower weight than those of OVX rats [21], which reveals that SDCP may also inhibit appetite. In spite of the significant differences in body weight, there was no significant change in the femur volume, length, or width in the OVX + SDCP and OVX + ALN groups when compared with the OVX group.

Bone turnover markers have been used to demonstrate the biological mechanism of SDCP on bone [25]. Serum ALP, OC, and OPN levels have previously been evaluated for bone formation [26]. ALP is critical in osteoid formation and mineralization, and serum total ALP is a widely applied marker of bone formation [27]. OC, a non-collagenous protein secreted by osteoblasts, participates in mineralization and $\mathrm{Ca}^{+}$homeostasis [28]. OPN has an important role to anchor osteoclasts to the mineral matrix of bones during the bone remodeling process [29]. An increase in the ALP level in OVX rats was also reported by another group [30]. Along with the increase in ALP, levels of OC and OPN, two other important indicators of bone formation, also significantly increased, which is consistent with a previous clinical report [31] and our previous study [32]. 

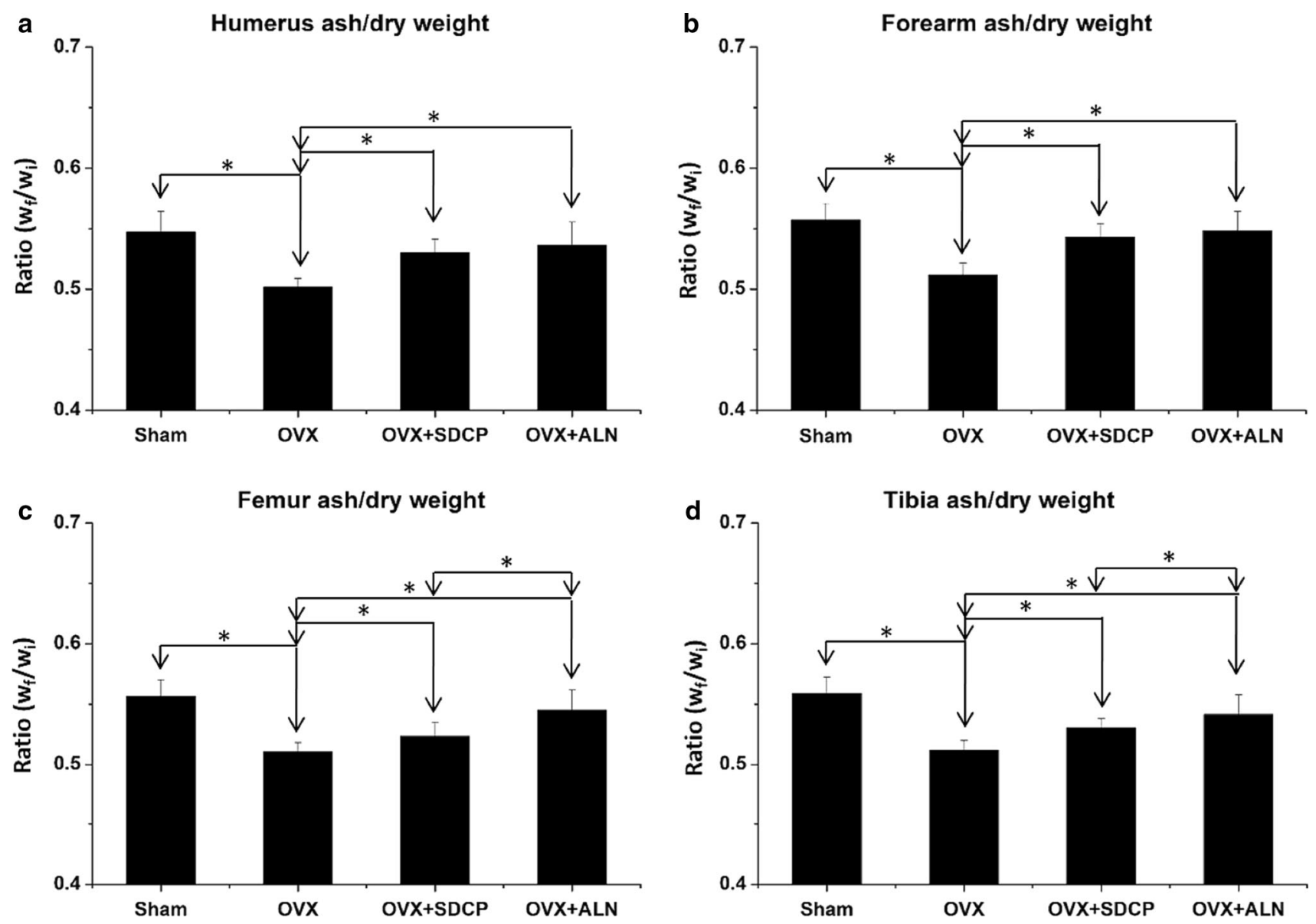

Fig. 4 Ratio of bone ash to dry weight $\left(\mathrm{w}_{\mathrm{f}} / \mathrm{w}_{\mathrm{i}}\right)$ of limbs decreased significantly post-ovariectomy (OVX group). Ingestion of SDCP and ALN significantly increased $\mathrm{w}_{\mathrm{f}} / \mathrm{w}_{\mathrm{i}}$ ratios of all limbs compared with OVX group. There were no significant differences in $\mathrm{w}_{\mathrm{f}} / \mathrm{w}_{\mathrm{i}}$ ratios of humerus and forearm among sham, OVX + SDCP, and OVX + ALN groups. However, $w_{f} / w_{i}$ ratios of femur and tibia in OVX + SDCP group were significantly lower than that of OVX + ALN group (both $p<0.05$ )

In spite of the increased levels of ALP, OC, and OPN, the bone resorption marker CTX-1 also increased significantly in the OVX rats. CTX-1 is a degradation product from C-terminal telopeptides of type 1 collagen, and the increase in CTX-1 represents an increase in the bone resorption rate [33]. In addition, levels of TRACP-5b, a phosphatase specifically expressed by bone-resorbing osteoclasts, decreased significantly. The amount of TRACP-5b represents the number of osteoclasts rather than osteoclast activity [34]. After OVX, serum TRACP-5b levels are expected to decrease because of the substantial bone loss, which causes a reduction in the number of osteoclasts in bone tissue. We observed increases in CTX-1 and decreases in TRACP-5b with a significant decrease in bone mass in the OVX rats. We hypothesize that the increase in serum bone formation markers in the OVX rats may be due to active osteoblasts trying to compensate for the bone loss caused by estrogen depletion. However, the speed of bone formation was still behind the bone resorption, thereby causing severe osteoporosis in the OVX rats. This hypothesis was supported by the histological sections of the distal femurs. The porosity of bony trabeculae increased, producing architectural discontinuity of cancellous bone in the OVX rats. In contrast, the serum ALP, OPN, OC, and CTX-1 levels in the OVX + SDCP group decreased significantly with increases in TRACP-5b and bone ash ratio. Since the TRACP-5b level and bone ash ratio show less change than the decreases in bone resorption markers, we assume that the inhibition of bone resorption is the predominant effect of SDCP. This finding reveals that SDCP ingestion inhibits bone resorption and slows bone turnover rate in the OVX rats. This result was further supported by the histological study of bones, which revealed an increase in trabecular bones and a decrease in porosity.

A previous study found that ALN treatment increased the secretion of endogenous PTH [35]. PTH regulates bone turnover to improve bone quality and accelerate fracture 
healing [36, 37]. In osteoporotic patients, PTH therapy can improve bone mineral density and reduce fracture rate [38]. In the present study, the rats administered with ALN had an increase in the serum PTH level. Moreover, the administration of SDCP also slightly increased the PTH level in the OVX rats, indicating that SDCP may have an effect similar to that of an intermittent administration of low-dose PTH. However, rats in the OVX + SDCP group had a lower serum PTH level than that in the OVX + ALN group, which reveals that SDCP has a weaker effect on increasing the secretion of endogenous nocturnal PTH. Regarding bone quality, the ingestion of SDCP decreased the bony porosity and increased the bone mineral content. Although the anti-osteoporosis effect of SDCP is weaker than that of $\mathrm{ALN}$, as determined from bone turnover markers and histological inspections, SDCP has better biocompatibility than that of ALN. The beneficial effects of SDCP were supported by the thickening of trabeculae, with restoration of interconnections, as shown in the histological studies.

Studies of pharmacokinetics and pharmacodynamics reveal that bisphosphonates are absorbed by paracellular transport throughout the gastrointestinal tract [39]. After absorption, bisphosphonates bind to the mineral component of bone, hydroxyapatite. Although bisphosphonates are generally believed to enter the extracellular space of the bone by paracellular transport, exactly how bisphosphonates transfer from the circulation to bone is not fully understood [40]. The elimination process of bisphosphonates from the skeleton is relatively slow since bisphosphonates are released during bone resorption. Renal excretion is the only route to eliminate bisphosphonates [39]. Although we cannot provide solid evidences in the present study, the metabolism and excretion of SDCP is supposed to be similar to that of bisphosphonates. Moreover, it is known that the elimination of pyrophosphate is also through renal excretion [41]. Further studies are required to clarify the pharmacokinetics and pharmacodynamics of SDCP.

\section{Conclusion}

No oral toxicity was found with the administration of SDCP in OVX rats in this long-term oral toxicity study. No clinical signs of toxicity, and no changes in food consumption, body weight, blood examination, necropsy, and histological inspection attributable to the oral administration of SDCP were found. The ingestion of SDCP decreased the bone turnover markers, as demonstrated by a significant decrease in the serum levels of ALP, OC, and OPN. SDCP inhibited bone resorption, as evidenced by the serum levels of TRACP-5b and CTX-1. Further, SDCP improved the architecture of trabecular bone, decreased the porosity, and increased the bone mineral content in long bones. The study indicates that SDCP inhibits bone resorption to increase bone mass in OVX rats. SDCP has potential in the management of osteoporosis.

Acknowledgements This study was supported by Shin Kong Wu Ho-Su Memorial Hospital (SKH-TMU-104-01).

\section{Compliance with Ethical Standards}

Conflict of interest The authors declare that they have no conflict of interest.

\section{References}

1. Russell, R. G., Espina, B., \& Hulley, P. (2006). Bone biology and the pathogenesis of osteoporosis. Current Opinion in Rheumatology, 18, S3-S10.

2. Takeda, S. (2008). Central control of bone remodeling. Journal of Neuroendocrinology, 20, 802-807.

3. Graham, R., \& Russell, G. (2006). Bisphosphonates. Annals of the New York Academy of Sciences, 1068, 367-401.

4. Boivin, G., \& Meunier, P. J. (2002). Effects of bisphosphonates on matrix mineralization. Journal of Musculoskeletal and Neuronal Interactions, 2, 538-543.

5. Jobke, B., Milovanovic, P., Amling, M., \& Busse, B. (2014). Bisphosphonate-osteoclasts: Changes in osteoclast morphology and function induced by antiresorptive nitrogen-containing bisphosphonate treatment in osteoporosis patients. Bone, 59, 37-43.

6. Chapurlat, R. D., \& Delmas, P. D. (2006). Drug insight: Bisphosphonates for postmenopausal osteoporosis. Nature Clinical Practice Endocrinology \& Metabolism, 2, 211-219.

7. Chapurlat, R. D. (2005). Clinical pharmacology of potent new bisphosphonates for postmenopausal osteoporosis. Treat Endocrinol, 4, 115-125.

8. Khapra, A. P., \& Rose, S. (2006). Drug injury in the upper gastrointestinal tract: Effects of alendronate. Gastrointestinal Endoscopy Clinics of North America, 16, 99-110.

9. Khosla, S., Burr, D., Cauley, J., Dempster, D. W., Ebeling, P. R., Felsenberg, D., et al. (2007). Bisphosphonate-associated osteonecrosis of the jaw: Report of a task force of the American Society for Bone and Mineral Research. Journal of Bone and Mineral Research, 22, 1479-1491.

10. Reid, D. M., Devogelaer, J. P., Saag, K., Roux, C., Lau, C. S., Reginster, J. Y., et al. (2009). Zoledronic acid and risedronate in the prevention and treatment of glucocorticoid-induced osteoporosis (HORIZON): a multicentre, double-blind, doubledummy, randomised controlled trial. Lancet, 373, 1253-1263.

11. Black, D. M., Delmas, P. D., Eastell, R., Reid, I. R., Boonen, S., Cauley, J. A., et al. (2007). Once-yearly zoledronic acid for treatment of postmenopausal osteoporosis. New England Journal of Medicine, 356, 1809-1822.

12. Lyles, K. W., Colón-Emeric, C. S., Magaziner, J. S., Adachi, J. D., Pieper, C. F., Mautalen, C., et al. (2007). Zoledronic acid and clinical fractures and mortality after hip fracture. New England Journal of Medicine, 357, 1799-1809.

13. Lee, J. H., Chang, B. S., Ryu, H. S., \& Lee, C. K. (2009). A 90-day subchronic toxicity study of beta-calcium pyrophosphate in rat. Drug and Chemical Toxicology, 32, 277-282.

14. Grover, L. M., Wright, A. J., Gbureck, U., Bolarinwa, A., Song, J., Liu, Y., et al. (2013). The effect of amorphous pyrophosphate on calcium phosphate cement resorption and bone generation. Biomaterials, 34, 6631-6637. 
15. Lin, F. H., Lin, C. C., Lu, C. M., Liu, H. C., Sun, J. S., \& Wang, C. Y. (1995). Mechanical properties and histological evaluation of sintered $\beta-\mathrm{Ca}_{2} \mathrm{P}_{2} \mathrm{O}_{7}$ with $\mathrm{Na}_{4} \mathrm{P}_{2} \mathrm{O}_{7} \cdot \beta-\mathrm{Ca}_{2} \mathrm{P}_{2} \mathrm{O}_{7}$ addition. Biomaterials, 16, 793-802.

16. Sun, J. S., Tsuang, Y. H., Liao, C. J., Liu, H. C., Hang, Y. S., \& Lin, F. H. (1997). The effects of calcium phosphate particles on the growth of osteoblast. Journal of Biomedical Materials Research, 37, 324-334.

17. Sun, J. S., Chang, W. H., Chen, L. T., Huang, Y. C., Juang, L. W., $\&$ Lin, F. H. (2004). The influence on gene-expression profiling of osteoblasts behavior following treatment with the ionic products of sintered beta-dicalcium pyrophosphate dissolution. Biomaterials, 25, 607-616.

18. Sun, J. S., Huang, Y. C., Lin, F. H., \& Chen, L. T. (2003). The effect of sintered dicalcium pyrophosphate on osteoclast metabolism: an ultrastructural study. Journal of Biomedical Materials Research Part A, 64, 616-621.

19. Kuo, Y. J., Sun, J. S., Rau, G., Chen, C. H., Tsai, T. H., \& Tsuang, Y. H. (2014). Better osteoporotic fracture healing with sintered dicalcium pyrophosphate (SDCP) treatment: A rat femoral fracture model. Journal of Histochemistry and Cytochemistry, 62, 565-576.

20. Lelovas, P. P., Xanthos, T. T., Thoma, S. E., Lyritis, G. P., \& Dontas, I. A. (2008). The laboratory rat as an animal model for osteoporosis research. Comparative Medicine, 58, 424-430.

21. Yang, K. C., Wang, C. C., Wu, C. C., Huang, T. Y., Chang, H. C., Chang, H. K., et al. (2010). Acute and subacute oral toxicity test of sintered dicalcium pyrophosphate on ovariectomized rats for osteoporosis treatment. Biomedical Engineering: Applications, Basis and Communications, 22, 169-176.

22. Kuo, Y. J., Tsuang, F. Y., Sun, J. S., Lin, C. H., Chen, C. H., Li, J. Y., et al. (2012). Calcitonin inhibits SDCP-induced osteoclast apoptosis and increases its efficacy in a rat model of osteoporosis. PLOS ONE, 7, e40272.

23. Milewicz, A., Tworowska, U., \& Demissie, M. (2001). Menopausal obesity-myth or fact? Climacteric, 4, 273-283.

24. Ogawa, K., Hori, M., Takao, R., \& Sakurada, T. (2005). Effects of combined elcatonin and alendronate treatment on the architecture and strength of bone in ovariectomized rats. Journal of Bone and Mineral Metabolism, 23, 351-358.

25. Markus, J. S. (2005). Biochemical markers of bone turnover part I: Biochemistry and variability. Clinical Biochemist Reviews, 26, 97-122.

26. Seibel, M. J., \& Woitge, H. W. (1999). Basic principles and clinical applications of biochemical markers of bone metabolism: Biochemical and technical aspects. Journal of Clinical Densitometry, 12, 299-321.

27. Garnero, P., \& Delmas, P. D. (1993). Assessment of the serum levels of bone alkaline phosphatase with a new immunoradiometric assay in patients with metabolic bone disease. Journal of Clinical Endocrinology and Metabolism, 77, 1046-1053.

28. Goldstone, A. P., Howard, J. K., Lord, G. M., Ghatei, M. A., Gardiner, J. V., \& Wang, Z. L. (2002). Leptin prevents the fall in plasma osteocalcin during starvation in male mice. Biochemical and Biophysical Research Communications, 295, 475-481.

29. Altintaş, A., Saruhan-Direskeneli, G., Benbir, G., Demir, M., \& Purisa, S. (2009). The role of osteopontin: A shared pathway in the pathogenesis of multiple sclerosis and osteoporosis? Journal of the Neurological Sciences, 276, 41-44.

30. Foucault, P., Foucault, M. H., Kucharewicz, B., Bureau, F., Alix, M., \& Drosdowsky, M. A. (1991). Value of the study of total alkaline phosphatases and bone isoenzyme in a population of subjects with osteoporosis. Annales de Biologie Clinique, 49, 477-481.

31. Kim, S. W., Park, D. J., Park, K. S., Kim, S. Y., Cho, B. Y., Lee, H. K., et al. (2005). Early changes in biochemical markers of bone turnover predict bone mineral density response to antiresorptive therapy in Korean postmenopausal women with osteoporosis. Endocrine Journal, 52, 667-674.

32. Yang, K. C., Yen, K. C., Huang, J. Y., Wu, C. C., \& Lin, F. H. (2011). Sintered dicalcium pyrophosphate decreases bone turnover rate in osteoporotic rat: A study on serum biochemical bone turnover markers. Biomedicine \& Aging Pathology, 1, 46-51.

33. Herrmann, M., \& Seibel, M. J. (2008). The amino- and carboxyterminal cross-linked telopeptides of collagen type I, NTX-I and CTXI: A comparative review. Clinica Chimica Acta, 393, 57-75.

34. Nishizawa, Y., Inaba, M., Ishii, M., Yamashita, H., Miki, T., Goto, H., et al. (2008). Reference intervals of serum tartrateresistant acid phosphatase type $5 \mathrm{~b}$ activity measured with a novel assay in Japanese subjects. Journal of Bone and Mineral Metabolism, 26, 265-270.

35. Greenspan, S. L., Holland, S., Maitland-Ramsey, L., Poku, M., Freeman, A., Yuan, W., et al. (1996). Alendronate stimulation of nocturnal parathyroid hormone secretion: A mechanism to explain the continued improvement in bone mineral density accompanying alendronate therapy. Proceedings of the Association of American Physicians, 108, 230-238.

36. Rubin, M. R., \& Bilezikian, J. P. (2005). Parathyroid hormone as an anabolic skeletal therapy. Drugs, 65, 2481-2498.

37. Ellegaard, M., Jørgensen, N. R., \& Schwarz, P. (2010). Parathyroid hormone and bone healing. Calcified Tissue International, 87, 1-13.

38. Peichl, P., Holzer, L. A., Maier, R., \& Holzer, G. (2011). Parathyroid hormone 1-84 accelerates fracture-healing in pubic bones of elderly osteoporotic women. Journal of Bone and Joint Surgery. American Volume, 93, 1583-1587.

39. Cremers, S., \& Papapoulos, S. (2011). Pharmacology of bisphosphonates. Bone, 49, 42-49.

40. Leu, C. T., Luegmayr, E., Freedman, L. P., Rodan, G. A., \& Reszka, A. A. (2006). Relative binding affinities of bisphosphonates for human bone and relationship to antiresorptive efficacy. Bone, 38, 628-636.

41. Miller, P. D., Jamal, S. A., Evenepoel, P., Eastell, R., \& Boonen, S. (2013). Renal safety in patients treated with bisphosphonates for osteoporosis: A review. Journal of Bone and Mineral Research, 28, 2049-2059. 